\title{
Late or variable onset
}

National Cancer Institute ( $\mathrm{NCl})$

\section{Source}

National Cancer Institute (NCI). Late or variable onset.

The state in which a genetic trait is expressed later in life or is expressed at no fixed time in a life history. 